\title{
Exploring protocol bias in airway microbiome studies: one versus two PCR steps and 16S rRNA gene region V3 V4 versus V4
}

Christine Drengenes ${ }^{1,2^{*}}$, Tomas M. L. Eagan ${ }^{1,2}$, Ingvild Haaland ${ }^{1,2}$, Harald G. Wiker ${ }^{2,3}$ and Rune Nielsen ${ }^{1,2}$

\begin{abstract}
Background: Studies on the airway microbiome have been performed using a wide range of laboratory protocols for high-throughput sequencing of the bacterial 16S ribosomal RNA (16S rRNA) gene. We sought to determine the impact of number of polymerase chain reaction (PCR) steps (1- or 2- steps) and choice of target marker gene region (V3 V4 and V4) on the presentation of the upper and lower airway microbiome. Our analyses included Illumina MiSeq sequencing following three setups: Setup 1 (2-step PCR; V3 V4 region), Setup 2 (2-step PCR; V4 region), Setup 3 (1-step PCR; V4 region). Samples included oral wash, protected specimen brushes and protected bronchoalveolar lavage (healthy and obstructive lung disease), and negative controls.

Results: The number of sequences and amplicon sequence variants (ASV) decreased in order setup1 $>$ setup2 $>$ setup3. This trend appeared to be associated with an increased taxonomic resolution when sequencing the V3 V4 region (setup 1) and an increased number of small ASVs in setups 1 and 2. The latter was considered a result of contamination in the two-step PCR protocols as well as sequencing across multiple runs (setup 1). Although genera Streptococcus, Prevotella, Veillonella and Rothia dominated, differences in relative abundance were observed across all setups. Analyses of beta-diversity revealed that while oral wash samples (high biomass) clustered together regardless of number of PCR steps, samples from the lungs (low biomass) separated. The removal of contaminants identified using the Decontam package in $\mathrm{R}$, did not resolve differences in results between sequencing setups.
\end{abstract}

Conclusions: Differences in number of PCR steps will have an impact of final bacterial community descriptions, and more so for samples of low bacterial load. Our findings could not be explained by differences in contamination levels alone, and more research is needed to understand how variations in PCR-setups and reagents may be contributing to the observed protocol bias.

Keywords: Microbiota, Contamination, Low biomass, Respiratory, 165 rRNA gene

\footnotetext{
*Correspondence: Christine.Drengenes@gmail.com

'Department of Thoracic Medicine, Haukeland University Hospital, Bergen, Norway

${ }^{2}$ Department of Clinical Science, Faculty of Medicine, University of Bergen, Bergen, Norway

Full list of author information is available at the end of the article
}

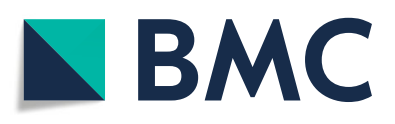

(c) The Author(s). 2021 Open Access This article is licensed under a Creative Commons Attribution 4.0 International License, which permits use, sharing, adaptation, distribution and reproduction in any medium or format, as long as you give appropriate credit to the original author(s) and the source, provide a link to the Creative Commons licence, and indicate if changes were made. The images or other third party material in this article are included in the article's Creative Commons licence, unless indicated otherwise in a credit line to the material. If material is not included in the article's Creative Commons licence and your intended use is not permitted by statutory regulation or exceeds the permitted use, you will need to obtain permission directly from the copyright holder. To view a copy of this licence, visit http://creativecommons.org/licenses/by/4.0/. The Creative Commons Public Domain Dedication waiver (http://creativecommons.org/publicdomain/zero/1.0/) applies to the data made available in this article, unless otherwise stated in a credit line to the data. 


\section{Background}

The bacterial airway microbiome has been studied using a wide range of protocols for high-throughput sequencing of the bacterial $16 \mathrm{~S}$ ribosomal RNA (16S rRNA) gene. Common to all amplicon based protocols is the application of the polymerase chain reaction (PCR) for $\mathrm{i}$ ) amplification of the target marker gene to be sequenced and ii) the addition of index sequences necessary for sample multiplexing. These steps can be performed in a single PCR or in two separate PCRs. No study has addressed whether the increased number of laboratory processing steps associated with a 2-step PCR protocol, will leave samples more vulnerable to bacterial DNA contamination from the laboratory than when following a 1-step PCR protocol. The inverse relationship between sample bacterial load and the impact of contamination has been well documented in the literature by others $[1$, 2] and ourselves [3]. Thus, we predicted that while samples with a high bacterial load (i.e. upper airway samples) would be able to buffer against protocol effects resulting from differences in contamination levels, samples with a low bacterial load (i.e. lower airway samples) would not be resistant to these effects.

In addition to number of PCR steps, sequencing protocols vary by choice of targeted marker gene region. Several different 16S rRNA gene variable regions have been targeted in studies of the lung microbiome, including V1 V2 [4, 5], V1 V3 [6-8], V3 V5 [7, 9-13], V3 [14, 15] and V4 [16-19]. Choice of target marker gene region has been limited by the short length of DNA that can be sequenced using the most common high-throughput sequencing technologies. The V4 region has increased in popularity as studies on estimates of alpha- [20] and beta- diversity [21] (i.e. measures of diversity within and between samples, respectively) and taxonomic assignments [22, 23] have collectively indicated that this site generates the most accurate descriptions. In addition, its relatively short length has allowed for the complete overlap of the forward and reverse sequencing read; advantageous because correction of sequencing errors is possible using the read with highest quality score [24]. The increased capacity of the MiSeq sequencer to sequence longer DNA sequences coupled with the development of novel denoising strategies (e.g. DADA2 [25]), has however led to an increased interest in the targeting of the longer V3 V4 region. It is however unclear how these results compare to earlier studies based on the shorter V4 region.

In the current study, we sought to i) evaluate the impact of bacterial DNA contamination when processing samples through protocols that vary in number of PCR steps (1- or 2-steps) and ii) determine the impact of choice of target marker gene region (V3 V4 vs V4) on the presentation of the upper and lower airway microbiome. To address these issues we processed samples of both high and low bacterial load through three library preparation setups varying in the number of PCR steps and target marker gene region: Setup 1 (2-step PCR; V3 V4 region), Setup 2 (2-step PCR; V4 region), Setup 3 (1-step PCR; V4 region). The upper airways were represented by oral wash (OW) samples and the lower airways by protected specimen brushes (PSB) and protected bronchoalveolar lavages (PBAL) collected by bronchoscopy. Negative control samples (NCS) consisting of saline used in the collection of all samples was processed together with the clinical samples for assessment of contamination.

\section{Results}

\section{Study participants}

The study included 23 subjects from the MicroCOPD study [26]. Subject characteristics are provided in Table 1.

\section{Number of sequences and amplicon sequence variants (ASVs)}

We began our analyses with a comparison of the number of sequences and amplicon sequence variants (ASVs) retained at each step when processing through the bioinformatic pipeline (Fig. 1). For sequencing setup 1, the procedural samples were dispersed across four sequencing runs (I-IV). For sequencing setups 2 and 3, two separate sequencing runs (one per setup) were conducted including all samples.

As the sequences were passed through the different bioinformatic filtering steps, the total number of sequences and ASVs across the three setups became more similar. Denoising in DADA2 (Fig. 1, step 1) resulted in the greatest decrease in sequence number. The greatest decrease in ASV number occurred after the removal of small ASVs, for which the number of sequences was calculated to be less than $0.005 \%$ of the total number of sequences on the same run (Fig. 1, step 3). The drop in ASV number was greatest for sequencing setups 1 and 2 , both of which are based on the longer 2-step PCR protocol.

After the final filtering step (Fig. 1, step 6), the number of ASVs was significantly higher for setup 1 compared to that observed for setups 2 and 3 . When we restricted analyses to samples from the largest sequencing run in setup 1 (14 participants, 56 samples) (Additional File 1: Fig. S.1.), the number of ASVs for setup 1 was now more comparable to that observed for setups 2 and 3 (Additional File 1: Fig. S.1., step 6). The higher number of ASVs still observed for setup 1, was expected due to the greater taxonomic resolution obtained when targeting a longer marker gene region (V3 V4). 
Table 1 Subject characteristics

\begin{tabular}{llll}
\hline & Controls & COPD & Asthma \\
\hline Subjects & 9 & 10 & 4 \\
Age, mean \pm SD years & $63.0 \pm 6.7$ & $68.2 \pm 5.2$ & $63.6 \pm 3.1$ \\
Men & $6(66.7 \%)$ & $8(80.0 \%)$ & $2(50.0 \%)$ \\
Current-smokers & $2(22.2 \%)$ & $1(10.0 \%)$ & 0 \\
Former-smokers & $5(55.6 \%)$ & $9(90.0 \%)$ & $3(75.0 \%)$ \\
Never-smokers & $2(22.2 \%)$ & 0 & $1(25.0 \%)$ \\
Smoker pack years, mean \pm SD years & $11.8 \pm 6.1$ & $25.2 \pm 8.1$ & $12.1 \pm 6.2$ \\
FEV 1 (\% predicted), mean \pm SD & $97.0 \pm 13.7$ & $72.6 \pm 23.2$ & $101.6 \pm 9.3$ \\
Inhaled corticosteroids & 0 & $2(20.0 \%)$ & $3(75.0 \%)$ \\
LABA & 0 & $3(30.0 \%)$ & $1(25.0 \%)$ \\
LAMA & 0 & $4(40.0 \%)$ & 0 \\
\hline
\end{tabular}

COPD Chronic obstructive pulmonary disease; FEV 1 Forced expiratory volume in $1 \mathrm{~s}$; LABA Long-acting beta-agonist; LAMA Long-acting muscarinic antagonist. 1

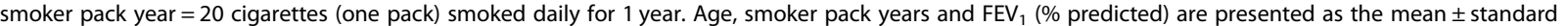
deviation.SD: standard deviation.

\section{Protocol effects on mock community sample}

The mock community sample HM-783D, consisting of genomic DNA from 20 different bacterial species (17 genera) was included on each sequencing run. For a detailed presentation of the mock community, see Additional File 7: Supplementary Methods. Because the protocols targeting different hypervariable regions result in different ASVs, we describe ASVs obtained for setup 1 (V3 V4 target) and setups 2 and 3 (V4 target), separately.

When following setup 1 across four sequencing runs, we obtained the following number of sequences and ASVs: run I: 128,413 (27 ASVs); run II: 109,709 (23 ASVs); run III: 110,492 (24 ASVs) and run IV: 84,909 (27 ASVs). As the number of sequences obtained for each run was similar, ASV numbers were also comparable across the four runs. While most genera were defined by a single ASV, genera Escherichia, Staphylococcus, Streptococcus, Clostridium and Rhodobacter were defined by multiple ASVs. The major ASVs attributed to each genus (i.e. those with the highest number of sequences) were the same across all four sequencing runs. For a detailed presentation of the ASVs observed in the mock community following setup 1 , see Additional File 2: Table S.1.

When following setups 2 and 3, we obtained 103,409 sequences (31 ASVs) and 120,073 sequences (23 ASVs), respectively. The genera Escherichia, Staphylococcus, Streptococcus, Clostridium and Neisseria were defined by multiple ASVs. The major ASVs attributed to each genus were the same in both setups 2 and 3. For a detailed presentation of the ASVs observed in the mock community following each setup, see Additional File 3: Table S.2. and Additional File 4:Table S.3.

A summary of the expected and observed taxonomic distribution in the mock community sample, obtained for each setup is presented in Fig. 2 and Table 2. We found that the three sequencing setups were for the most part equally efficient at recovering high abundant mock community members. Sequencing setup 3, was least efficient at recovering the low abundant members. Across all setups, we observed an increase in the relative abundances of genera Escherichia and Staphylococcus and a significant decrease in Rhodobacter compared to that expected. All setups generated low abundant ASVs that did not match to any of the expected taxa in the mock community (i.e contaminants). Because the mock community sample was included on each of the four sequencing runs I-IV performed following setup 1, we were also able to show that mock community sequencing is reproducible.

\section{Protocol effects on contamination profiles}

Our working hypothesis linked protocol bias to differences in susceptibility to laboratory contamination. We therefore proceeded with an examination of the average top 20 ASVs found in NCS. Because the same DNA extracts were processed through each of the three setups, any observed differences in taxonomic distribution would be attributed to library preparation steps (post DNA extraction). We also examined PCR water samples included on each sequencing run. In contrast to NCS, this later sample reflects contamination introduced during library preparation steps without interference from contaminating DNA introduced from the DNA extraction kit. ASVs obtained for setups 2 and 3, targeting the $\mathrm{V} 4$ region and the single setup targeting the V3 V4 region are described separately.

The average top 20 ASVs observed in NCS in setups 2 and 3, are presented in Fig. 3. The samples were dominated by many of the same taxa, and most of these taxa were defined by the same ASVs. The Decontam package (method $=$ either, threshold $=0.5)$ applied downstream of 


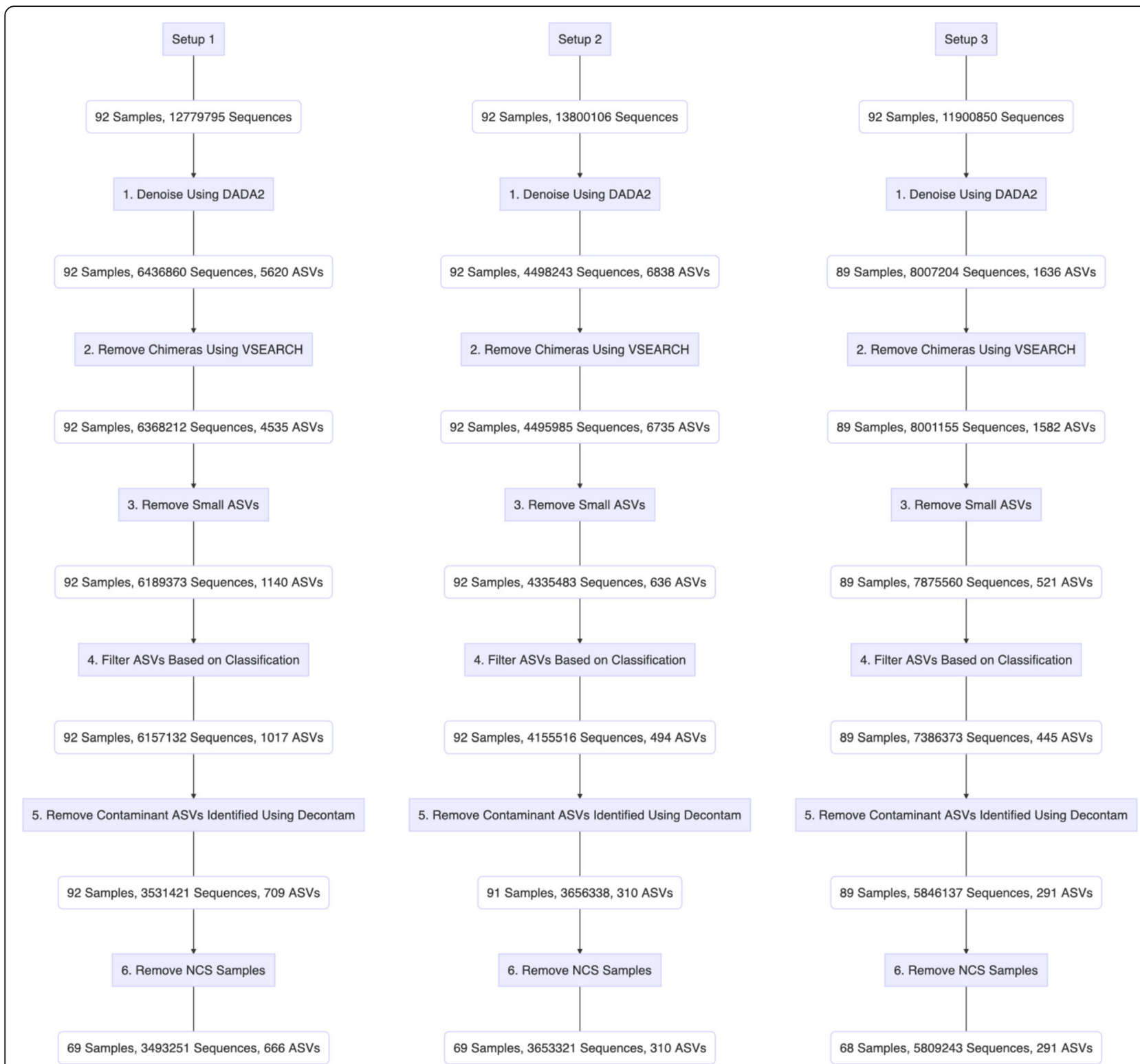

Fig. 1 Comparison of the number of sequences and amplicon sequence variants (ASVs), retained at each bioinformatic filtering step for procedural samples (PSB, PBAL, OW, NCS) collected from 23 participants ( $n=92$ samples). Setup 1 (2-step PCR; V3 V4 region), Setup 2 (2-step PCR; V4 region), Setup 3 (1-step PCR; V4 region)

the presented data identified the majority of the top 20 ASVs presented in NCS as contaminants. Exceptions included both ASVs mapping to the genus Streptococcus (in line with our previous findings [3]) (using NCBI blastn these ASVs were determined to be Streptococcus oralis (06f825b512d903b9230e1a55d87359ee) and Streptococcus thermophilus (fd496fd32dc8c08ade2e8b6c9d8ee13d) and the single ASV mapping to the family Pasteurellaceae.

The distribution of ASVs in NCS (Fig. 3) differed the most between setups 2 and 3 for an ASV belonging to the family Enterobacteriaceae (mapped to Escherichia using NCBI blastn), with a significant increase observed in samples sequenced by setup 3 (0.02\% observed for setup 2 and $29.34 \%$ observed for setup 3). These findings were in accordance with the results from the mock community analysis (Fig. 2), for which the same Escherichia ASV was also found at higher levels in the mock community sample sequenced by setup 3 (22.54\% observed for setup 2 and $32.90 \%$ observed for setup 3 ). Its relatively high abundance in the mock community processed through setup 2 compared to NCS was expected as the Escherichia genus defined by this ASV constituted $21.91 \%$ of the expected mock community profile; i.e. 


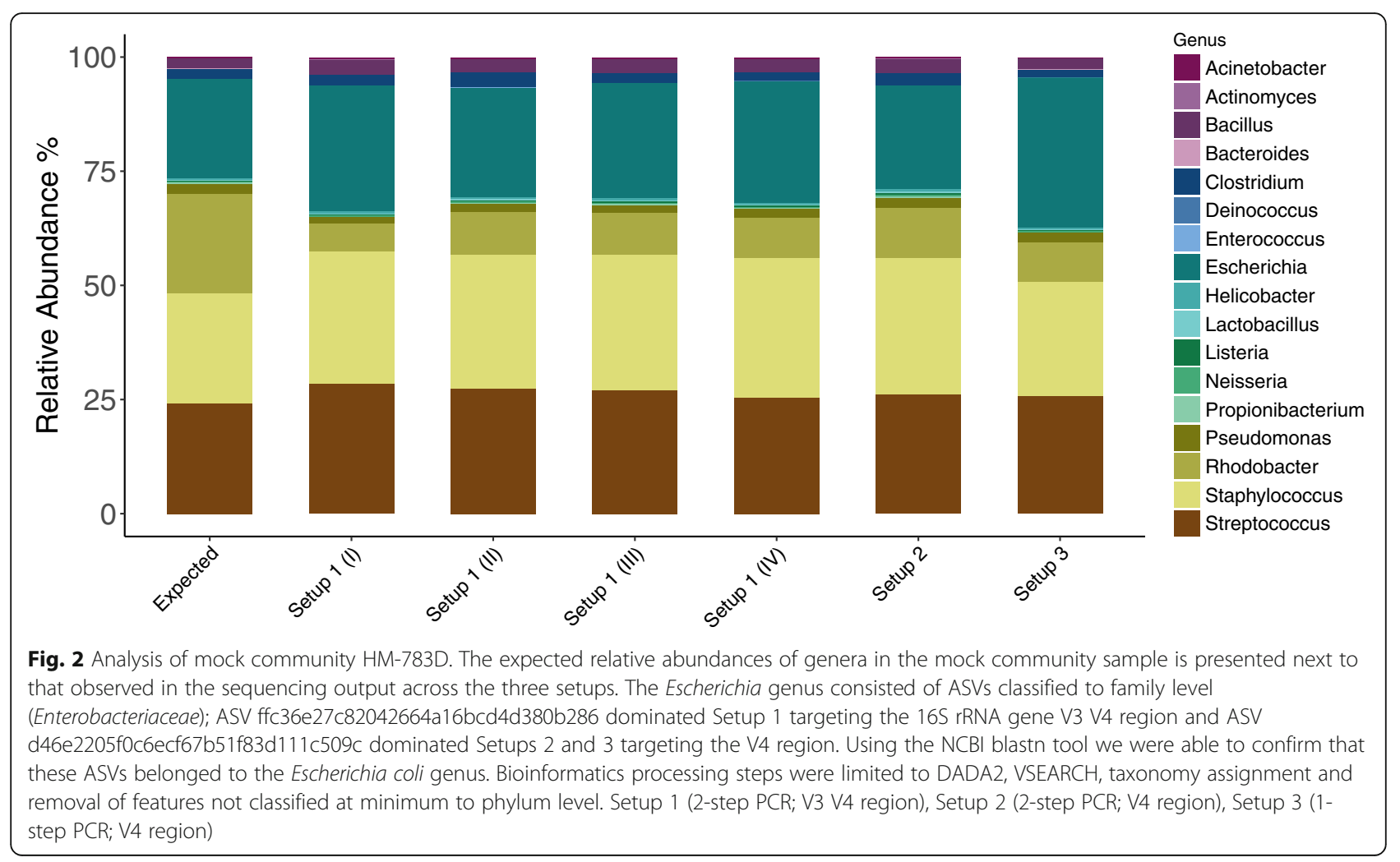

Table 2 Expected and observed relative abundance (\%) of genera in mock community sample HM-783D. Setup 1 (2-step PCR; V3 V4 region); Setup 2 (2-step PCR; V4 region); Setup 3 (1-step PCR; V4 region)

\begin{tabular}{|c|c|c|c|c|c|c|c|}
\hline Genera & Expected & $\begin{array}{l}\text { Setup } 1 \\
\text { (I) }\end{array}$ & $\begin{array}{l}\text { Setup } 1 \\
\text { (II) }\end{array}$ & $\begin{array}{l}\text { Setup } 1 \\
\text { (III) }\end{array}$ & $\begin{array}{l}\text { Setup } 1 \\
\text { (IV) }\end{array}$ & Setup 2 & Setup 3 \\
\hline Escherichia & 21.91 & 27.68 & 23.99 & 25.20 & 26.65 & 22.54 & 32.90 \\
\hline Rhodobacter & 21.91 & 5.98 & 9.52 & 9.23 & 8.94 & 11.00 & 8.77 \\
\hline Staphylococcus & 24.10 & 29.02 & 29.27 & 29.66 & 30.56 & 29.88 & 24.98 \\
\hline Streptococcus & 24.12 & 28.51 & 27.39 & 27.03 & 25.38 & 26.20 & 25.81 \\
\hline Bacillus & 2.19 & 3.38 & 2.86 & 2.95 & 2.85 & 3.15 & 2.49 \\
\hline Clostridium & 2.19 & 2.18 & 3.28 & 2.19 & 1.88 & 2.64 & 1.69 \\
\hline Pseudomonas & 2.19 & 1.44 & 1.68 & 1.75 & 1.93 & 2.12 & 2.02 \\
\hline Acinetobacter & 0.22 & 0.32 & 0.29 & 0.33 & 0.30 & 0.29 & 0.12 \\
\hline Helicobacter & 0.22 & 0.36 & 0.49 & 0.44 & 0.38 & 0.61 & 0.26 \\
\hline Lactobacillus & 0.22 & 0.22 & 0.20 & 0.23 & 0.24 & 0.35 & 0.18 \\
\hline Listeria & 0.22 & 0.33 & 0.33 & 0.30 & 0.32 & 0.37 & 0.26 \\
\hline Neisseria & 0.22 & 0.24 & 0.31 & 0.30 & 0.27 & 0.43 & 0.39 \\
\hline Propionibacterium & 0.22 & 0.13 & 0.22 & 0.18 & 0.15 & 0.29 & 0.00 \\
\hline Actinomyces & 0.02 & 0.01 & 0.01 & 0.00 & 0.00 & 0.01 & 0.00 \\
\hline Bacteroides & 0.02 & 0.02 & 0.00 & 0.03 & 0.02 & 0.04 & 0.02 \\
\hline Deinococcus & 0.02 & 0.02 & 0.04 & 0.03 & 0.02 & 0.03 & 0.02 \\
\hline Enterococcus & 0.02 & 0.03 & 0.02 & 0.03 & 0.02 & 0.02 & 0.00 \\
\hline Other & 0.00 & 0.13 & 0.11 & 0.10 & 0.09 & 0.03 & 0.08 \\
\hline
\end{tabular}




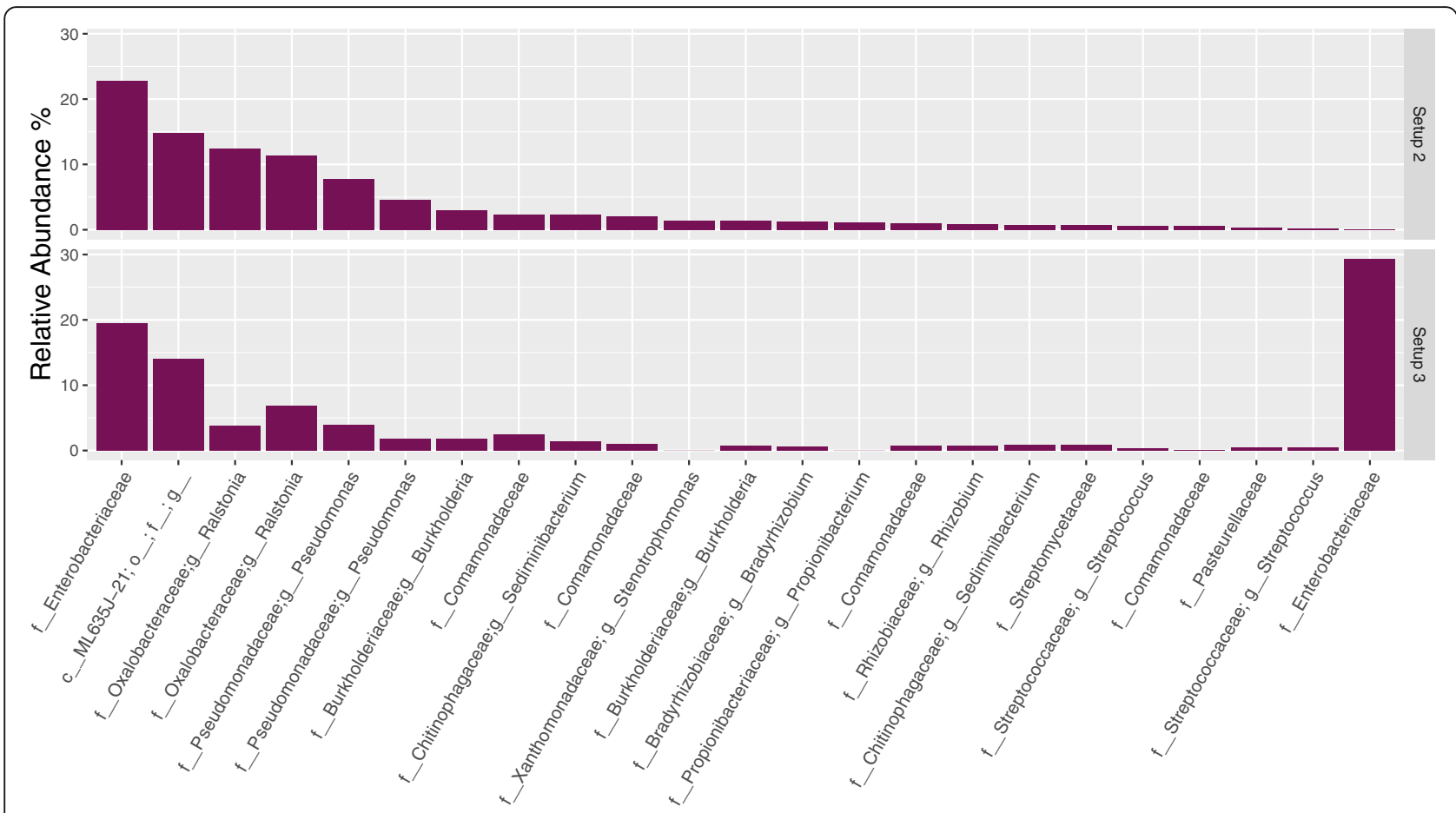

Fig. 3 Comparison of the 20 most abundant amplicon sequence variants (ASVs) observed in negative control samples (NCS) after sequencing following setups 2 and 3. Taxa presented according to decreasing abundance for ASVs observed following setup 2. Bioinformatic processing steps were performed up the removal of contaminants identified using Decontam. Taxonomic rank is described using prefixes (c_: class, o_ order, f_: family, g_: genus). Setup 2 (2-step PCR; V4 region); Setup 3 (1-step PCR; V4 region). Data is presented as the average relative abundance. Data unrarefied

for this sample the ASV represented both a contaminant and a non-contaminant.

We proceeded with a comparison of the taxonomic distribution in PCR water samples sequenced following setups 2 and 3 (Table 3 ). A relatively low number of sequences and ASVs were obtained (setup 2: 178 sequences (10 ASVs); setup 3: 130 sequences (6 ASVs)). Importantly, the dominating ASV (35.38\%) found in the PCR water samples sequenced following setup 3, was the same ASV mapping to Escherichia discussed above. The

Table 3 Relative abundance (\%) of ASVs observed in PCR water samples in setups 2 and 3

\begin{tabular}{|c|c|c|c|}
\hline ASV & Lowest Classification & Setup 2 & Setup 3 \\
\hline 06f825b512d903b9230e1a55d87359ee & f__Streptococcaceae; g__Streptococcus & 35.39 & 20.77 \\
\hline ddfd49f939f92958b1ec816741055348 & f__Oxalobacteraceae; g__Ralstonia; s__ & 12.36 & 0.00 \\
\hline 394eda29c886632f514dd94b58381186 & f__Pasteurellaceae & 8.99 & 0.00 \\
\hline d32e579b3ae7b2aae8d5bf9f027c29af & f__Comamonadaceae & 8.99 & 0.00 \\
\hline 5648dccee530d68ceb3e4d7d22cf8756 & f_Pseudomonadaceae; g_Pseudomonas & 7.87 & 0.00 \\
\hline 4f5efd25dacb5d639316e7291ff6ff8b & f__Neisseriaceae; g__Neisseria & 7.87 & 7.69 \\
\hline 85c44c83eddc5d3028261a1000b7d0e1 & f__Gemellaceae & 5.62 & 0.00 \\
\hline 923f521b9cf313f1f95c9367e09bbc1c & f__Veillonellaceae; g_Veillonella; s_dispar & 5.62 & 12.31 \\
\hline dcba105f35d8ebc9e22269c7491ad3a7 & f__Xanthomonadaceae; g__Stenotrophomonas; s_geniculata & 5.06 & 0.00 \\
\hline df8456a1abbfb4c8a2c450b44378d4cb & f__Actinomycetaceae; g__Actinomyces; s__ & 2.25 & 0.00 \\
\hline d46e2205f0c6ecf67b51f83d111c509c* & f_Enterobacteriaceae & 0.00 & 35.38 \\
\hline edc9e5c16e40aff1eadce6597940f08f & f__Streptococcaceae; g__Streptococcus; s__ & 0.00 & 13.85 \\
\hline 65d43491988bfe557da4d86a5ba25dae & f__Staphylococcaceae; g_Staphylococcus & 0.00 & 10.00 \\
\hline
\end{tabular}

*Escherichia ASV also observed to differentiate mock community samples and NCS in setups 2 and 3. Bioinformatic processing steps were performed up until the removal of contaminants identified using Decontam. Taxonomic rank is described using prefixes (f_: family, g_: genus, s_: species) 


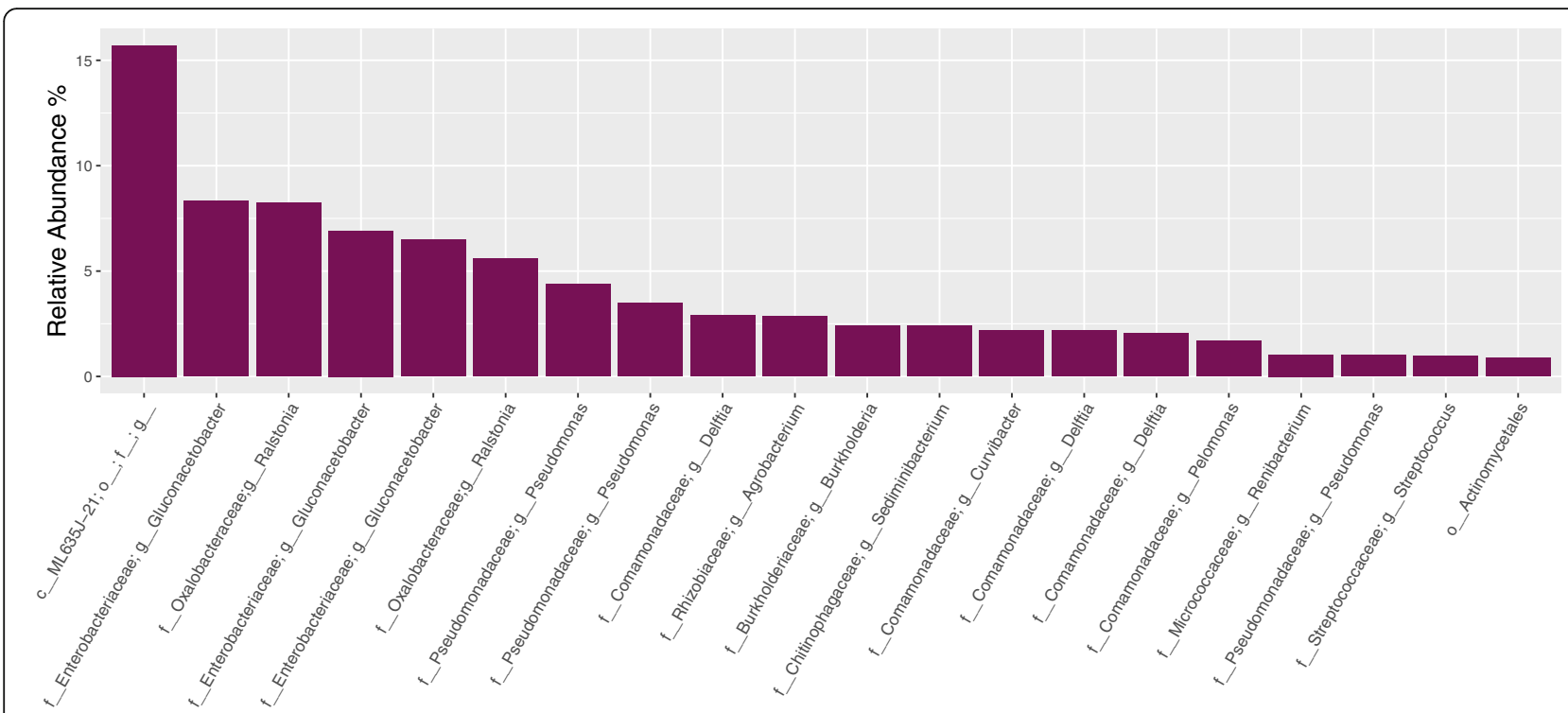

Fig. 4 The 20 most abundant amplicon sequence variants (ASVs) observed in negative control samples (NCS) after sequencing following setup 1. Multiple ASVs mapped to genera Gluconacetobacter, belonging to familiy Enterobacteriaceae (cummulative 22\%). Bioinformatic processing steps were performed up until the removal of contaminants identified using Decontam. Taxonomic rank is described using prefixes ( $c$ __ class, o__: order, f__ family, g_: genus). Data is presented as the average relative abundance. Data unrarefied

same ASV was not found in the PCR water sample sequenced by setup 2. Together these findings indicate that the Escherichia ASV is a contaminant introduced during steps of library preparation using a reagent that is exclusive to setup 3 .

We next looked at the average top 20 ASVs observed in NCS when sequencing following setup 1 (Fig. 4). The taxonomic profiles obtained after sequencing the longer V3 V4 region resulted in greater taxonomic resolution compared to that observed when sequencing the V4 region in setups 2 and 3 . Whereas the three ASVs belonging to the family Enterobacteriaceae classified down to genus level Gluconacetobacter in setup 1, the Enterobacteriaceae ASVs classified no lower than to family level in setups 2 and 3 (Fig. 3). The cumulative average relative abundance of the three ASVs mapping to Gluconacetobacter when following setup $1(22 \%)$ was however the same as that found for the single ASV mapping to the family Enterobacteriaceae when following setup 2 (23\%). Thus, for these two setups, the contamination profiles were similar although greater resolution was obtained when sequencing a longer target gene region in setup 1 (V3 V4).

\section{Protocol effects on procedural samples}

We next compared the sequencing output obtained for the procedural samples sequenced following each of the three setups. Because we suspected that any differences observed between sequencing setups could be explained by differences in susceptibility to laboratory contamination, comparisons were made both before and after the removal of contaminants identified in Decontam (Fig. 1, Step 5).

Before the removal of Decontam contaminants (Fig. 5), we found that across all three sequencing setups, procedural samples (OW, PSB, PBAL) were dominated by many of the same taxa. The most prominent taxa averaged across all samples in order of decreasing relative abundance were genera Streptococcus, Prevotella, Veillonella and Rothia. We interpreted these as representative of the authentic airway microbiota based on the growing body of literature for which these same taxa have been consistently observed in airways.

Several less abundant taxa for which we interpreted as contaminants, based on their dominance in NCS were also observed in the data. We previously learned that ASVs attributed to the family Enterobacteriaceae dominated the NCS and that an ASV mapping to Escherichia had a discriminating impact on NCS and mock communities processed through setup 3. We were therefore particularly interested in understanding whether Enterobacteriaceae would also have a discriminating impact on procedural samples processed through the different sequencing setups. Across all three sequencing setups we found that the levels of Enterobacteriaceae was highest in samples from the lower airways (PSB $>$ PBAL) and nearly undetected in OW samples (Fig. 5). The higher levels of Enterobacteriaceae in PSB samples compared to PBAL, was expected as less sample volume was used as input to the DNA extraction protocol $(450 \mu \mathrm{l}$ PSB vs $1800 \mu \mathrm{l}$ PBAL) thereby securing a lower bacterial load in PSB compared to PBAL. Across all sample types, the 


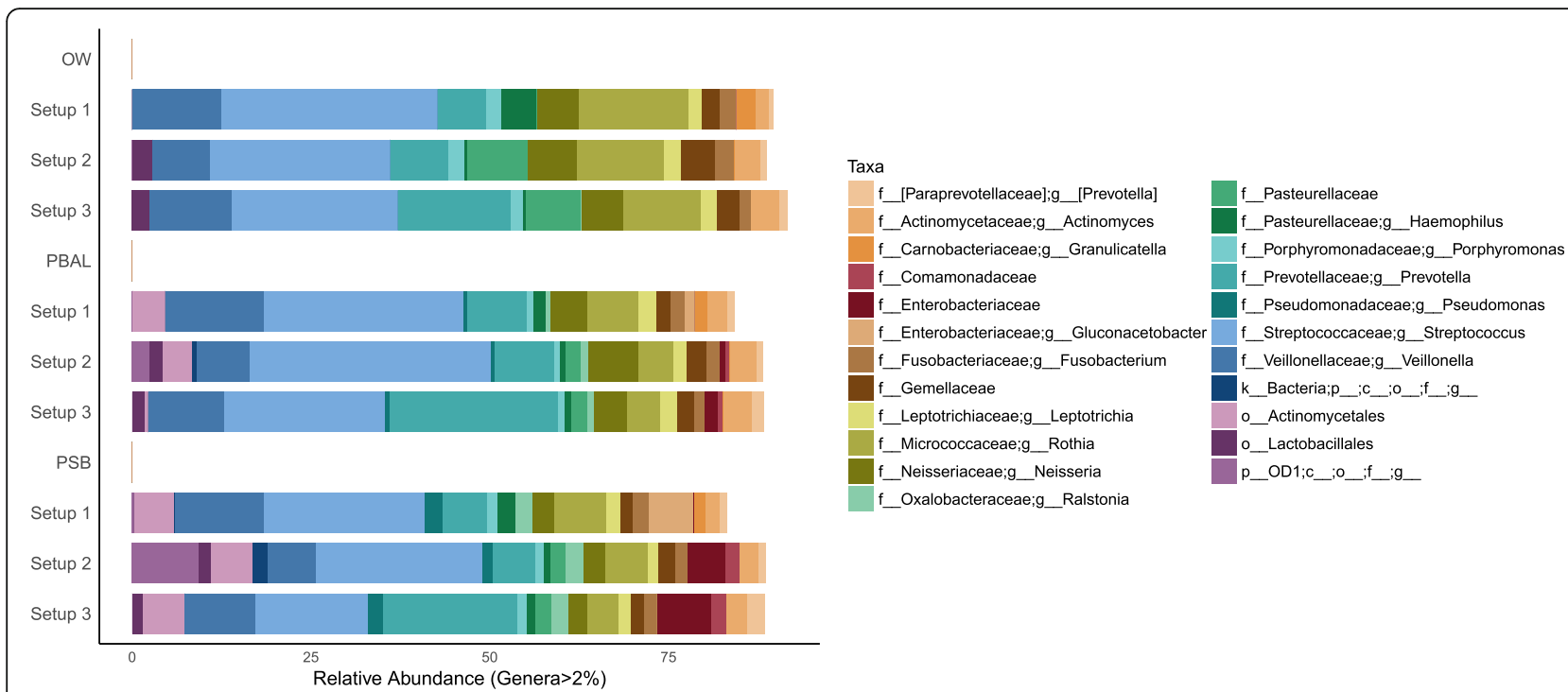

Fig. 5 Taxonomic distribution obtained for procedural samples before the removal of Decontam contaminants. ASVs attributed to the family Enterobacteriaceae, had dominated the NCS across all setups. In the procedural samples, Enterobacteriaceae was observed with the following relative abundances in setups 2 and 3: setup 2 (OW: 0\%; PBAL: 0.83\%; PSB: 5.23\%); setup 3 (OW: 0.01\%; PBAL: 1.87\%; PSB: 7.51\%). ASVs attributed to the genus Gluconacetobacter within the family Enterobacteriaceae was observed in procedural samples with the following relative abundances in setup 1 (OW: 0\%; PBAL: 1.42\%; PSB: 6.32\%). Samples with fewer than 1000 sequences had been omitted from the analyses leaving the following number of samples in each setup: Setup 1 (OW: $n=22$; PBAL: $n=23$; PSB: $n=23$ ); Setup 2 (OW: $n=23 ;$ PBAL: $n=23 ;$ PSB: $n=23$ ); Setup 3 (OW: $n=23$; PBAL: $n=21$; PSB: $n=22$ ). Setup 1 (2-step PCR; V3 V4 region); Setup 2 (2-step PCR; V4 region); Setup 3 (1-step PCR; V4

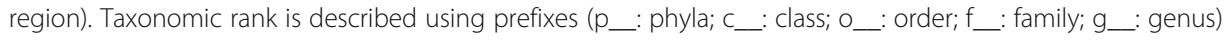

relative abundance of Enterobacteriaceae was highest when sequencing following setup 3; this was also in accordance with our results when sequencing the mock community and likely due to the additional Escherichia contamination introduced during library preparation following setup 3 (Fig. 3). The relative abundance of Enterobacteriaceae per sample is provided in the Additional File 5: Table S.4. By analysis of beta-diversity using the unweighted UniFrac metric, we were able to confirm that there was greater overlap or similarity between the bacterial communities found in NCS and procedural samples from the lungs when sequencing following setup 3 (Additional File 6: Fig. S.2).

After the removal of Decontam contaminants, the less abundant taxa that we predicted as representative of contaminants had been filtered out (Fig. 6). Although the dominating taxa across all samples were now mainly expected core airway microbiota members, the relative abundances of these taxa still varied across the three setups.

A direct comparison of the bacterial communities recovered when sequencing by a 1 or 2 step PCR protocol was achieved by analysis of beta-diversity (using the unweighted UniFrac metric) on samples processed through each of setups 2 and 3. Before the removal of Decontam contaminants, OW and NCS clustered together regardless of whether they had been processed through setups 2 or 3 (Fig. 7). The samples from the lungs however clustered separately according to the protocol for which they were processed. When Decontam contaminants were removed, the samples from the lungs processed by setups 2 and 3 became more similar in bacterial community composition, as indicated by a greater degree of overlap in PCoA space (Fig. 8). The separation of the lower airway samples based on the setup for which they were processed was however still apparent. This indicated that mechanisms related to the low bacterial load, other than differences in contamination were driving the observed protocol bias. For analysis of beta-diversity using the weighted UniFrac metric before and after removal of Decontam contaminants, see Additional File 8: Fig. S.3 and Additional File 9: Fig. S.4, respectively.

\section{Discussion}

We have shown that choice of library preparation protocol for high-throughput amplicon-based sequencing of the 16S rRNA gene (1-step PCR vs 2-step PCR) will have an impact on final bacterial community descriptions for airway samples - and more so for samples of low bacterial load. Differences observed when sequencing the different target regions (V3 V4 and V4) appeared to be relatively small in comparison, and mainly attributed to differences in taxonomic resolution. Using bioinformatic filtering parameters, we were able to reduce but not completely remove the differences in sequencing output observed for the three sequencing setups: Setup 1 (2- 


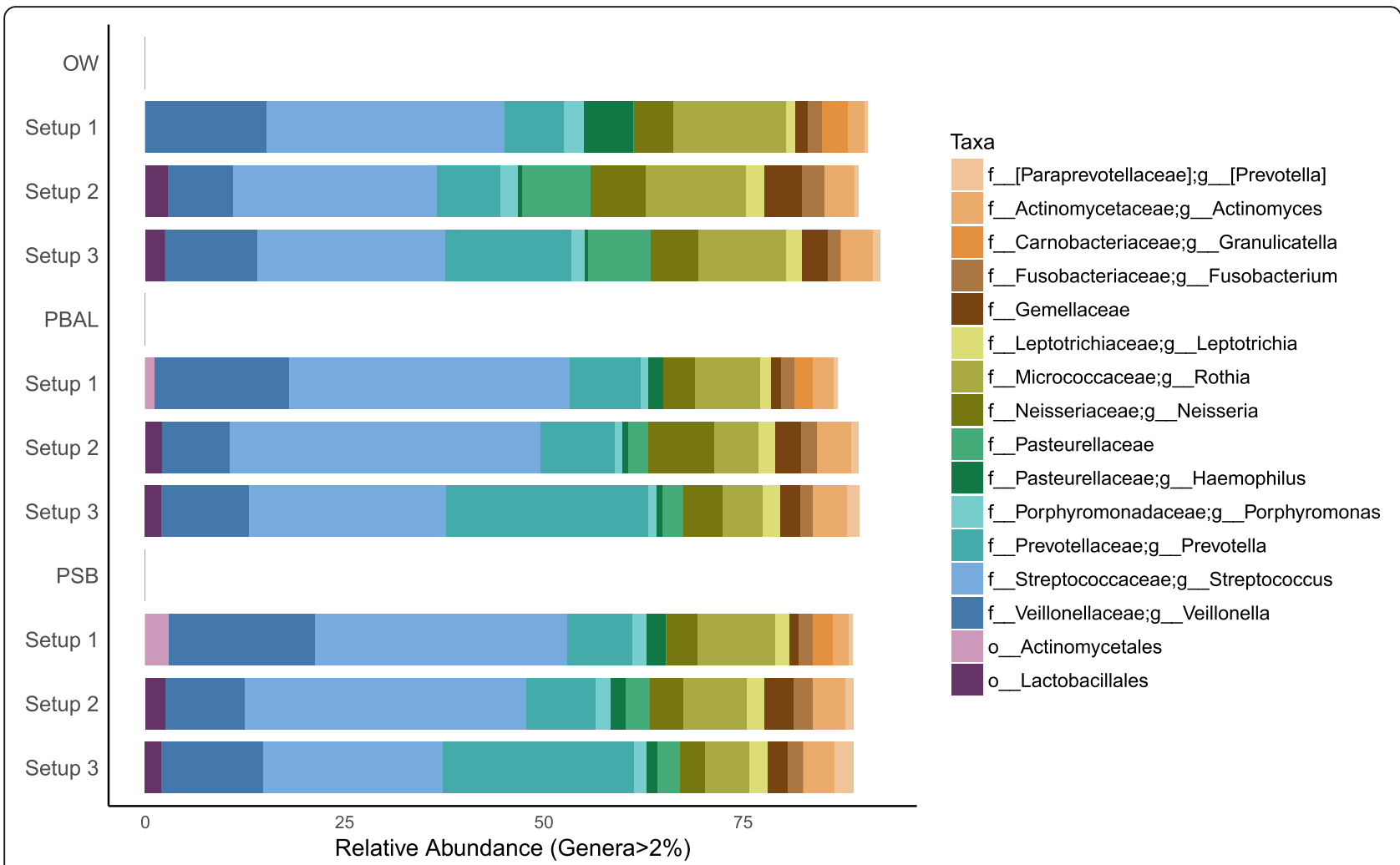

Fig. 6 Taxonomic distribution obtained for procedural samples after the removal of Decontam contaminants. Samples with fewer than 1000 sequences were omitted. Number of samples in each setup: V3V4 protocol A (OW: $n=22 ;$ PBAL: $n=22 ;$ PSB: $n=21)$, V4 protocol A (OW: $n=23$; PBAL: $n=22$; PSB: $n=20)$; V4 protocol B (OW: $n=23$; PBAL: $n=21$; PSB: $n=21$ ). Setup 1 (2-step PCR; V3 V4 region); Setup 2 (2-step PCR; V4 region); Setup 3 (1-step PCR; V4 region). Taxonomic rank is described using prefixes (p_: phyla; c_: class; O__: order; $f$ _: family; g_: genus)

step PCR; V3 V4), Setup 2 (2-step PCR; V4) and Setup 3 (1-step PCR; V4). We propose that protocol bias in studies of the lung microbiome are related not only to differences in susceptibility to contamination but also to less understood (and largely ignored) mechanisms of PCR bias.

Beginning with a comparison of the number of sequences and ASVs retained at each bioinformatic processing step, we gained insight into the differences in the sequencing output generated for each of the three setups. We found that the removal of small ASVs resulted in the greatest decrease in total ASV number across all three setups - with greatest impact on data generated from the two sequencing setups based on the 2-step PCR protocol (setup 1 and 2). Our interpretation was that the small ASVs likely represent low abundant contamination and that the observed higher frequencies in data generated when processing through longer laboratory workflows was as predicted. Interestingly, this filtering step was originally recommended for filtering out spurious operational taxonomic units (OTUs) derived from PCR and sequencing error [27], and therefore not regarded as necessary after denoisning to ASVs [28]. The total number of ASVs after the removal of small
ASVs, was still markedly higher when sequencing was performed following setup 1 , for which samples were spread across four different sequencing runs. We can expect contamination profiles to vary across sequencing runs, thereby adding to the number of ASVs in the data set, and we therefore interpreted the higher number of ASVs as contamination that had not been filtered out. When analyses were conducted on the subset of samples sequenced on the same run, we still observed a slight increase in ASV count in setup 1; this likely attributed to the greater taxonomic resolution obtained when sequencing a larger gene region. Based on the raw sequencing data, the take home message is therefore that researchers need to pay particular attention to small ASVs when making comparisons across datasets sequenced following different protocols. The observed inflation of ASVs when sequencing across multiple sequencing runs also needs to be accounted for.

By sequencing of a mock community sample, we were able to show that the three sequencing setups were for the most part equally efficient at recovering the high abundant mock community members. For reasons that are unclear to us, we found that sequencing setup 3 , was least efficient at recovering the low abundant members. Together with 


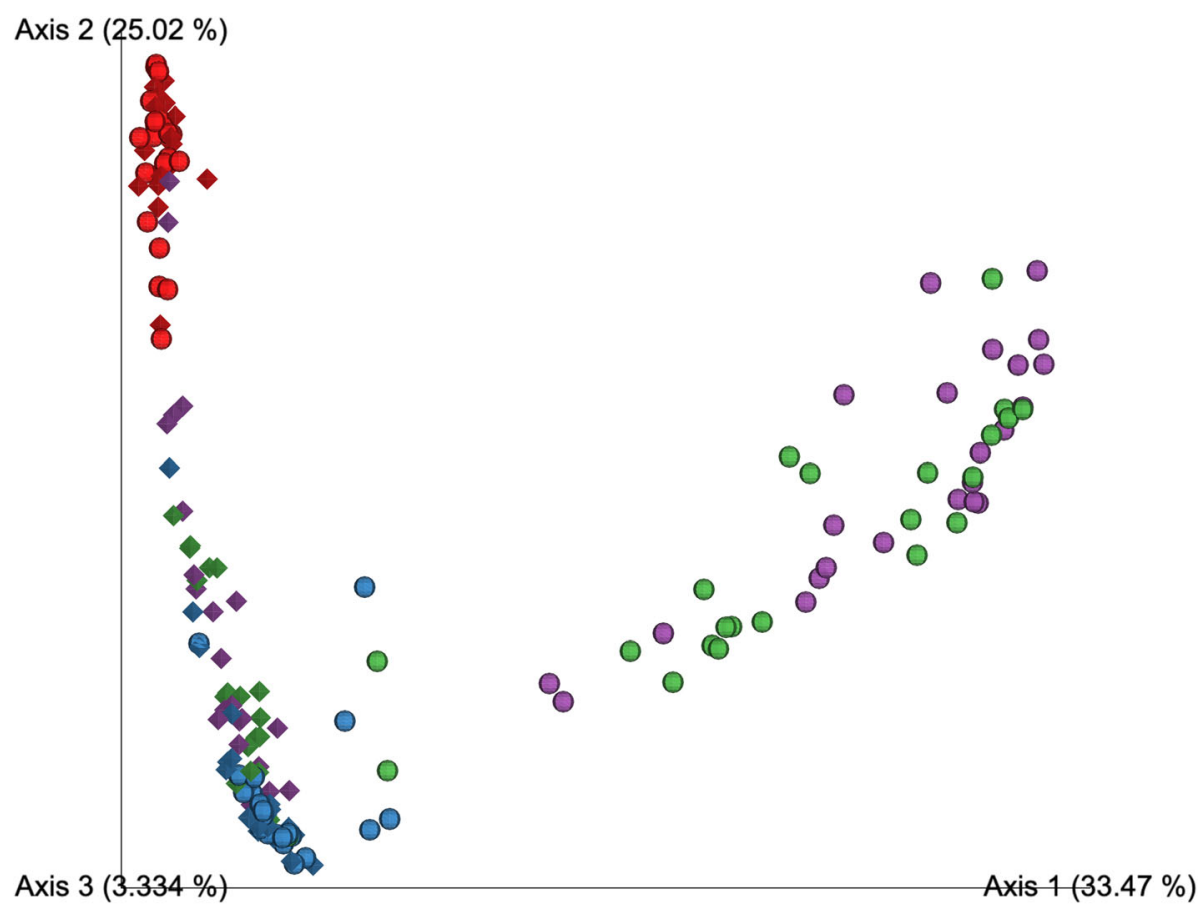

Fig. 7 Principal coordinates analysis on unweighted UniFrac distances for procedural samples sequenced following setup 2 (sphere) and 3 (diamond) before the removal of Decontam contaminants. Rarefaction depth: 1066 sequences. Setup 2 samples include OW: $n=23 ;$ PBAL: $n=23$; PSB: $n=23$; NCS: $n=21$ and setup 3 samples include OW: $n=23$; PBAL: $n=21$; PSB: $n=22 ;$ NCS: $n=18$. Oral Wash (OW): blue; Protected bronchoalveolar lavage (PBAL): green; Protected specimen brushes (PSB): purple; Negative control samples (NCS): red. Setup 2 (2-step PCR; V4 region), Setup 3 (1-step PCR; V4 region)

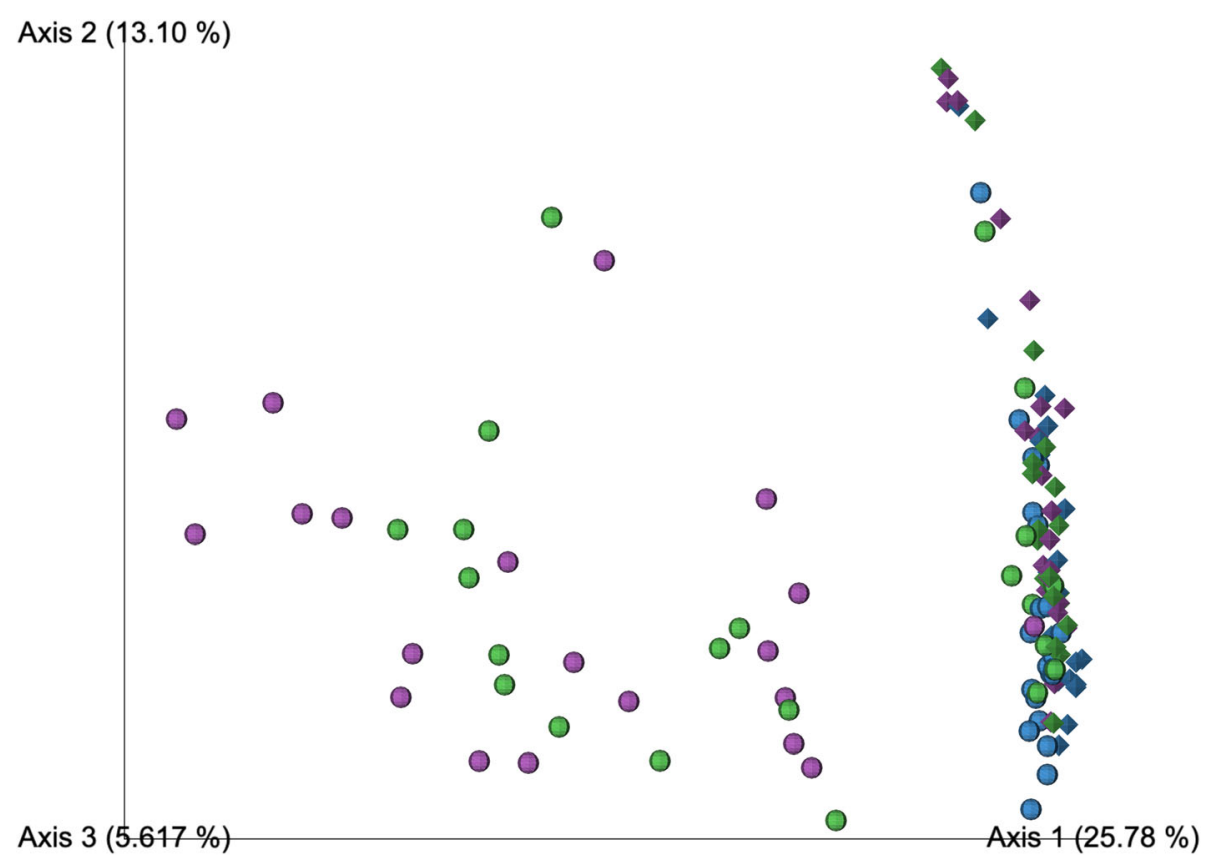

Fig. 8 Principal coordinates analysis on unweighted Unifrac distances for procedural samples sequenced following setup 2 (sphere) and 3 (diamond) after the removal of Decontam contaminants. Rarefaction depth: 1139 sequences. Setup 2 samples include OW: $n=23 ;$ PBAL: $n=22$; PSB: $n=20$ and setup 3 samples include OW: $n=23$; PBAL: $n=21$; PSB: $n=21$. Oral Wash (OW): blue; Protected bronchoalveolar lavage (PBAL): green; Protected specimen brushes (PSB): purple. Setup 2 (2-step PCR; V4 region), Setup 3 (1-step PCR; V4 region) 
the observation that the total number of ASVs recovered following setup 3 was lower than for Setups 1 and 2, we concluded that the 1 step-PCR protocol may be less apt for detecting rare but potentially significant taxa $[29,30]$. Berry et al. [31] also compared sequencing data generated when processing samples through PCR protocols that differed in the number of PCR steps (1-step PCR vs 2-step PCR). In accordance with our findings, they observed reduced richness when processing samples through the 1step PCR protocol. Thus, it could be that although the 1step PCR protocol may generate data less influenced by small contaminating ASVs, measures of alpha diversity may be underestimated.

To further explore the potential impact of contamination, we compared the contamination profiles (based on NCS) obtained for the three sequencing setups. We were surprised to find that the NCS samples processed through setup 3 were dominated by an ASV mapping to Escherichia coli (family Enterobacteriaceae). It was unexpected because we have previously traced the main source of contamination in the MicroCOPD study to the DNA extraction kit [3]. Because the same DNA extracts were used as input into the sequencing setup 3, we expected that the lower number of laboratory processing steps compared to setups 1 and 2, would secure a contaminant profile representative of that introduced during DNA extraction. We however learned that a contaminant introduced during library preparation was enough to overwhelm the contamination profile of the entire sequencing run. We immediately suspected that the DNA polymerase, manufactured in Escherichia coli and used exclusively in the PCR amplification step when sequencing following setup 3, was the main contamination source. Our findings emphasize the fact that researchers must be meticulous in their choice of PCR reagents and also aware of these effects when comparing data generated using different protocols.

We have previously determined the sample bacterial load for the samples included in the current study, and estimated that contaminants will represent $10-50 \%$ of the sequencing output for lower airway samples when sequencing by setup 1 [3]. We found that the Enterobacteriaceae family represented less than $10 \%$ of the taxonomy profiles for the procedural samples in all three setups and recognized that a significant fraction of the contaminants, were likely also represented by small ASVs and other taxa. For a more accurate assessment of the impact of contamination, we therefore also relied on the Decontam $\mathrm{R}$ package [32] for the identification of contaminants. We predicted that if contamination was the main distinguishing factor causing the separation in sequencing output across sequencing setups, the removal of Decontam contaminants would close this gap. By analysis of unweighted Unifrac distances in PCoA space, both before and after the removal of Decontam contaminants, we observed that while the high biomass OW samples clustered together, the low biomass samples from the lungs (PBAL,PSB) separated according to the setup 2 or 3 , for which they had been processed. We concluded that factors related to bacterial load, other than contamination must also be contributing to the observed protocol bias.

The polymerase chain reaction (PCR) lies at the core of all amplicon-based sequencing protocols. The impact of PCR related bias (i.e. all mechanisms that may lead to the preferential amplification of particular sequences or taxa) on studies involving samples holding a low bacterial load is however not well understood. This despite that recent papers as well as research dating back even two decades has documented that PCR related bias appears to increase with decreasing template DNA concentration [1, 33-35]. Kennedy et al. [35] observed that bacterial community profiles of replicate soil samples decreased in similarity after sample dilution. The authors attributed these observations to an increased impact of stochastic fluctuations in PCR amplifications at lower bacterial loads. Biesbroek et al. [1] observed an increase in Firmicutes and decrease in Bacteriodetes across a serially diluted saliva sample, but were unable to explain the direct mechanism behind their observations. Our study contributes to the literature addressing these issues by demonstrating that samples of high bacterial load (OW) appear to be able to buffer against protocol bias (i.e. differences in number of PCR steps), while samples of low bacterial load (PSB, PBAL) are directly impacted. More research is needed in order to understand the extent to which these mechanisms are responsible for our observations.

The results presented in the current study have several important implications. Because the upper respiratory tract represents both i) a major potential source of contamination under sampling and ii) the main source community for the lung microbiota, most studies include representative samples from this site (e.g. OW samples) $[4,17,19,36,37]$. Our findings demonstrate that the observed overlap between the bacterial communities of the upper and lower respiratory tract may be protocol dependent. Of concern is also that similar community descriptions obtained for upper respiratory tract samples across protocols may mistakenly be interpreted as evidence that datasets are comparable also for lower respiratory tract samples. Our findings also lead us to question the conclusions made in studies where similar PCR reagents have been used. Dickson et al. [12] have for example suggested that Escherichia coli may be a significant lung pathogen that has previously gone undetected using culture-based techniques. Our results open for interpreting the bacterium as a contaminant 
introduced with the recombinant DNA polymerase used in the PCR.

We acknowledge that there are limitations to our study. First of all, we did not collect control samples from the bronchoscope working channel used to obtain PBAL and PSB samples. We have however conducted such analyses in our previous publication [3], and shown the DNA extraction kit is the main contamination source for setup 1. For the current work, we focus on PCR protocol steps post-DNA extraction. Because the same DNA extracts were used as input to the three library preparation setups being compared, contamination from earlier steps of the pipeline would affect samples similarly in the different setups. Another limitation is that library preparation and sequencing for each setup was not repeated, and assessement of reliability therefore limited. However, our analyses of the mock community sample, for which was included on all three setups (and processed four times through setup 1), indicated that valid comparisons could be made across setups.

\section{Conclusion}

Our findings show that choice of protocol for library preparation and sequencing (1- or 2- steps of PCR) will have an impact on the analyses of the airway microbiome. Upper airway samples (high biomass) were less impacted than lower airway samples (low biomass), indicating that protocol bias is related to sample biomass. This did not appear to be associated with differences in contamination levels when following a longer or shorter protocol, but rather to mechanisms related to the PCR, for which more research is required. These methodological limitations likely explain the variable conclusions across studies of the airway microbiome (e.g. for comparisons of upper and lower airway samples). Differences in targeted amplicon region (16S rRNA gene V3 V4 versus V4) did not appear have a great impact on final bacterial community descriptions, although greater taxonomic resolution was observed when targeting the longer V3 V4 region.

\section{Methods}

\section{Study samples}

The 23 study subjects were chosen from the Bergen COPD Microbiome Study (short name "MicroCOPD") for representation of both healthy $(n=9)$ and diseased (asthma $(n=4)$, COPD $(n=10))$ states. Out of the 350 study subjects included in the MicroCOPD study (with samples dispersed across over 30 sequencing runs), the subset of subjects included in the current investigation were chosen in order to minimize the spread of samples across multiple runs. Details on the MicroCOPD study design and bronchoschopy procedures have been previously published [26]. The MicroCOPD study was approved by the regional ethical committee (REK-Vest, case \# 2011-1307), and all subjects signed written informed consent.

In brief, voluntary bronchoscopies were performed on adult subjects (with and without obstructive lung disease) recruited from Western Norway between 2013 and 2015, at the Department of Thoracic Medicine, Haukeland University Hospital. Subjects were examined in the stable state and were not to have received antibiotics at minimum 2 weeks prior to the procedure. Samples collected under each procedure included the first and second fraction of $2 \times 50 \mathrm{~mL}$ protected (through a sterile inner catheter passed through the scope channel) bronchoalveolar lavage (PBAL1 and PBAL2) from the right middle lobe, three protected specimen brushes sampled from the right lower lobe (PSB), an oral wash (OW) sample, and a negative control sample (NCS) taken from the sterile bottle of phosphate buffered saline directly; the same fluid used for BAL sampling, OW, and dissolution of the PSBs.

We also included a mock community sample, obtained through BEI Resources NIAID, NIH as part of the Human Microbiome Project: Genomic DNA from Microbial Mock Community B (Staggered, Low Concentration), v5.2L, for 16S rRNA Gene Sequencing, HM783D.

\section{Bacterial DNA extraction}

Bacterial DNA extraction was performed first by treatment with lytic enzymes mutanolysin, lysozyme and lysostaphin (all from Sigma-Aldrich, St. Louis, MO, USA) and subsequently by processing through the Fast DNA Spin Kit (MP Biomedcals, LLC, Solon, OH, USA) following the manufacturer's instructions. The sample volume used as input into the DNA extraction protocol varied with sample type; $450 \mu \mathrm{l}$ for PSB and NCS and $1800 \mu \mathrm{l}$ for OW and PBAL.

\section{Library preparation for MiSeq sequencing}

We processed the same DNA extracts through three different library preparation setups for MiSeq sequencing of the bacterial 16S rRNA marker gene: Setup 1 (2-step PCR; 16S rRNA gene region V3 V4); Setup 2 (2-step PCR; 16S rRNA gene region V4); Setup 3 (1-step PCR; $16 \mathrm{~S}$ rRNA gene region V4). Setups 1 and 2, were based on the 2-step PCR protocol described in the Illumina 16S Metagenomic Sequencing Library Preparation guide (Part no. 15044223 Rev. B). In the first PCR, the $16 \mathrm{~S}$ rRNA gene regions V3 V4 (setup 1) and V4 (setup 2) were targeted using primers (gene specific sequences are underlined):

Setup 1:

5' -TCGTCGGCAGCGTCAGATGTGTATAAGAGA

CAGCCTACGGGNGGCWGCAG-3' and.

5' -GTCTCGTGGGCTCGGAGATGTGTATAAGAG ACAGGACTACHVGGGTATCTAATCC-3'. 
Setup 2:

5'TCGTCGGCAGCGTCAGATGTGTATAAGAGA

CAGGTGCCAGCMGCCGCGGTAA3' and.

5'GTCTCGTGGGCTCGGAGATGTGTATAAGAG

ACAGGGACTACHVGGGTWTCTAAT3'.

PCR cycling was performed with an initial cycle at $95^{\circ} \mathrm{C}$ for $3 \mathrm{~min}$ followed by 45 cycles of $95^{\circ} \mathrm{C}$ for $30 \mathrm{~s}$, $55^{\circ} \mathrm{C}$ for $30 \mathrm{~s}$ (setup 1$) / 50^{\circ} \mathrm{C}$ (setup 2 ), $72{ }^{\circ} \mathrm{C}$ for $30 \mathrm{~s}$ and a final extension cycle at $72^{\circ} \mathrm{C}$ for $5 \mathrm{~min}$. In the second PCR (8 cycles), index sequences were added to the ends of the amplicons generated in the first PCR, using primers from the Nextera XT Index Kit (Illumina Inc., San Diego. CA, USA). Amplifications were performed using the Kappa HiFi HotStart ReadyMix (KAPA Biosystems, USA). Setup 3 was based on the 1-step PCR protocol described in Kozich et al. [24], with modifications (see Additional File 7: Supplementary Methods). The primers used targeted the 16S rRNA gene region V4 and consisted of both gene specific sequences (underlined) and index sequences $(\mathrm{N})$ :

Setup 3: 5'AATGATACGGCGACCACCGAGATCTA CACNNNNNNNNTATGGTAATTGTGTGCCAGCMG CCGCGGTAA3'.

\section{5'CAAGCAGAAGACGGCATACGAGATNNNNNN NNAGTCAGTCAGCCGGACTACHVGGG TWTCTAAT3'.}

PCR cycling was performed with an initial cycle at $95^{\circ} \mathrm{C}$ for $2 \mathrm{~min}$ followed by 45 cycles of $95^{\circ} \mathrm{C}$ for $20 \mathrm{~s}$, $55^{\circ} \mathrm{C}$ for $15 \mathrm{~s}, 72^{\circ} \mathrm{C}$ for $5 \mathrm{~min}$ and a final extension cycle at $72^{\circ} \mathrm{C}$ for $5 \mathrm{~min}$. Amplifications were performed using the recombinant DNA polymerse Accuprime Pfx Super Mix (Thermo Fisher Scientific, USA).

\section{Bioinformatics}

General Steps. Sequences were processed using plugin tools available within the Quantitative Insights Into Microbial Ecology (QIIME2) bioinformatic package (release 2019.1). Two fastq-files per sample (demulitiplexed, paired-end reads) were imported into the QIIME2 environment. Using the dada2 denoise-paired plugin i) primer sequences and low quality bases at read-ends were trimmed off, ii) paired-end reads were joined, iii) chimeras discarded and iv) amplicon sequence variants (ASVs) inferred [25, 28]. Additional chimera filtering was performed using the vsearch uchime-denovo plugin [38]. ASVs with fewer sequences than $0.005 \%$ of the total number of sequences and ASVs not found in at least two samples were then discarded [27]. Taxonomy was assigned using the feature-classifier classify-sklearn plugin together with a Naïve Bayes classifier that had been pre-trained on the full-length Greengenes 13_8 99\% OTU reference database (available on qiime2.org). ASVs classified as mitochondria, chloroplasts or archaea were discarded together with classifications that ended above the phylum level. Contaminant ASVs identified using the Decontam package in $\mathrm{R}$ were then discarded [32]. The Decontam method "either" (threshold $=0.5$ ) was chosen based on our previous work [3]. As the study samples were found across multiple sequencing runs, bioinformatics processing of samples was performed in batches according to run number. Samples not included in the study, but present on the same run were also included in the pipeline to optimize performance of run specific algorithms (e.g. DADA2 and Decontam). Analyses. Analysis on taxonomic composition was performed in Excel on ASV tables generated at various stages of the bioinformatic pipeline. Analyses on procedural samples (PSB, PBAL, OW) were performed on the ASV table processed through all general steps described above. Analyses on the top 20 ASVs found in NCS and in PCR water controls, were based on the ASV table processed through all steps in the pipeline except removal of contaminants identified in Decontam. For analyses on mock community samples, processing steps were limited to DADA2, VSEARCH and removal of ASVs not classified at minimum to phylum level. Analyses of beta-diversity were conducted using PCoA on unweighted UniFrac distances. The unweighted UniFrac metric scores samples with bacterial communities found at similar positions within the phylogenetic tree, as more similar than samples with bacterial communities found at different positions within the tree. The (dis)similarity between samples is visualized in principal coordinates of analysis (PCoA) space, with samples similar in bacterial composition plotted closer together. The unweighted UniFrac metric was chosen to ensure that the less abundant ASVs would have equal impact on the clustering pattern as the high abundant ASVs.

\section{Supplementary Information}

The online version contains supplementary material available at https://doi. org/10.1186/s12864-020-07252-z.

Additional file 1: Fig. S1. Comparison of the number of sequences and amplicon sequence variants (ASVs) retained at each bioinformatic filtering step for procedural samples (PSB, PBAL, OW, NCS) collected from 14 participants $(n=56)$.

Additional file 2: Table S1. The table presents an overview of the sequence count per ASV obtained after V3 V4 sequencing of mock community sample HM-783D following setup 1.

Additional file 3: Table S2. The table presents an overview of the sequence count per ASV obtained after V4 sequencing of mock community sample HM-783D following setup 2.

Additional file 4: Table S3. The table presents an overview of the sequence count per ASV obtained after V4 sequencing of mock community sample HM-783D following setup 3.

Additional file 5: Table S4. The table presents an overview of the relative abundance of ASVs mapping to Enterobacteriaceae per sample across all sample types and setups. 
Additional file 6: Fig. S2. Principal coordinates analysis on unweighted UniFrac distances for procedural samples sequenced following each setup before the removal of Decontam contaminants.

Additional file 7: Supplementary Methods. The file provides a detailed description of the mock community HMD 783-D, protocols for sequencing.

Additional file 8: Fig. S3. Principal coordinates analysis on weighted Unifrac distances for procedural samples sequenced following setup 2 (sphere) and 3 (diamond) before the removal of Decontam contaminants.

Additional file 9: Fig. S4. Principal coordinates analysis on weighted UniFrac distances for procedural samples sequenced following setup 2 (sphere) and 3 (diamond) after the removal of Decontam contaminants.

\section{Abbreviations}

ASV: Amplicon sequence variant; COPD: Chronic obstructive pulmonary disease; EMP: Earth microbiome project; HMP: Human microbiome project: NCS: Negative control sample; OTU: Operational taxonomic unit; OW: Oral wash; PBAL: Protected bronchoalveolar lavage; PSB: Protected specimen brushes; QIIME: Quantitative insights into microbial ecology

\section{Acknowledgements}

The authors wish to thank Marit Aardal, Kristina Apalseth, Hildegunn Bakke Fleten, Ane Aamli Gagnat, Tuyen Thi Van Hoang, Gunnar Husebø, Tharmini Kalananthan, Kristel Knudsen, Sverre Lehmann, Lise Østgård Monsen, Eli Nordeide, Randi Sandvik, Øistein Svanes for their contributions in the data collection and/or analyses.

\section{Authors' contributions}

TME, RN, IH and HGW participated in the planning and collection of procedural samples in the MicroCOPD study. HGW and CD planned the sequencing analyses. CD performed DNA extraction, library preparation for sequencing, bioinformatics analyses and drafted the manuscript. RN and TME participated in bioinformatics analyses and drafting of the manuscript. All authors participated in the revision of the manuscript and approved the final version for publication.

\section{Funding}

The MicroCOPD study was funded by unrestricted grants and fellowships from Helse Vest, Bergen Medical Research Foundation, the Endowment of timber merchant A. Delphin and wife through the Norwegian Medical Association and GlaxoSmithKline through the Norwegian Respiratory Society. The funding bodies had no role in the design of the study, data collection and analysis, interpretation of data, or in writing the manuscript.

\section{Availability of data and materials}

Fastq files and metadata are available at https://doi.org/10.5061/dryad. $8 c z 8 \mathrm{w} 9 \mathrm{gnt}$. Protocols and laboratory materials used in the MicroCOPD study are available at dx.doi.org/10.17504/protocols.io.2sygefw.

\section{Ethics approval and consent to participate}

The study was approved by the Regional Committees for Medical and Health Research Ethics (REK-Nord, case \# 2011/1307) and was conducted in accordance with the Declaration of Helsinki. All study subjects signed informed consent forms.

\section{Consent for publication}

Not applicable.

\section{Competing interests}

CD, HGW: The authors declare that they have no competing interests. TME: Reports bursary from Boehringer Ingelheim for educational meetings within the last three years, unrelated to the current study. RN: Reports grants from GlaxoSmithKline, during the conduct of the study; grants from Boehringer Ingelheim, grants and personal fees from AstraZeneca, grants from Novartis, grants from Boehringer Ingelheim, personal fees from GlaxoSmithKline, outside the submitted work.

\section{Author details}

'Department of Thoracic Medicine, Haukeland University Hospital, Bergen, Norway. ${ }^{2}$ Department of Clinical Science, Faculty of Medicine, University of Bergen, Bergen, Norway. ${ }^{3}$ Department of Microbiology, Haukeland University Hospital, Bergen, Norway.

Received: 10 June 2020 Accepted: 18 November 2020

Published online: 04 January 2021

\section{References}

1. Biesbroek G, Sanders EAM, Roeselers G, Wang X, Caspers MPM, Trzciński K, et al. Deep sequencing analyses of low density microbial communities: working at the boundary of accurate microbiota detection. PLoS One. 2012; 7:e32942.

2. Salter SJ, Cox MJ, Turek EM, Calus ST, Cookson WO, Moffatt MF, et al. Reagent and laboratory contamination can critically impact sequence-based microbiome analyses. BMC Biol. 2014;12:87.

3. Drengenes C, Wiker HG, Kalananthan T, Nordeide E, Eagan TML, Nielsen R. Laboratory contamination in airway microbiome studies. BMC Microbiol. 2019:19:187

4. Charlson ES, Bittinger K, Haas AR, Fitzgerald AS, Frank I, Yadav A, et al. Topographical continuity of bacterial populations in the healthy human respiratory tract. Am J Respir Crit Care Med. 2011;184:957-63.

5. Einarsson GG, Comer DM, Mcllreavey L, Parkhill J, Ennis M, Tunney MM, et al. Community dynamics and the lower airway microbiota in stable chronic obstructive pulmonary disease, smokers and healthy non-smokers. Thorax. 2016;71:795-803.

6. Erb-Downward JR, Thompson DL, Han MK, Freeman CM, McCloskey L, Schmidt LA, et al. Analysis of the lung microbiome in the "healthy" smoker and in COPD. PLoS One. 2011;6. https://doi.org/10.1371/journal.pone. 0016384.

7. Morris A, Beck JM, Schloss PD, Campbell TB, Crothers K, Curtis JL, et al. Comparison of the respiratory microbiome in healthy nonsmokers and smokers. Am J Respir Crit Care Med. 2013;187:1067-75.

8. Beck JM, Schloss PD, Venkataraman A, Twigg H, Jablonski KA, Bushman FD, et al. Multicenter comparison of lung and Oral microbiomes of HIV-infected and HIV-uninfected individuals. Am J Respir Crit Care Med. 2015;192:1335-44.

9. Bassis CM, Erb-Downward JR, Dickson RP, Freeman CM, Schmidt TM, Young $V B$, et al. Analysis of the upper respiratory tract microbiotas as the source of the lung and gastric microbiotas in healthy individuals. mBio. 2015;6: e00037-15.

10. Dickson RP, Erb-Downward JR, Freeman CM, Walker N, Scales BS, Beck JM, et al. Changes in the lung microbiome following lung transplantation include the emergence of two distinct Pseudomonas species with distinct clinical associations. PLoS One. 2014;9. https://doi.org/10.1371/journal.pone.0097214.

11. Dickson RP, Erb-Downward JR, Prescott HC, Martinez FJ, Curtis JL, Lama VN, et al. Cell-associated bacteria in the human lung microbiome. Microbiome. 2014;2:28.

12. Dickson RP, Erb-Downward JR, Prescott HC, Martinez FJ, Curtis JL, Lama VN, et al. Analysis of culture-dependent versus culture-independent techniques for identification of Bacteria in clinically obtained Bronchoalveolar lavage fluid. J Clin Microbiol. 2014:52:3605-13.

13. Venkataraman A, Bassis CM, Beck JM, Young VB, Curtis JL, Huffnagle GB, et al. Application of a neutral community model to assess structuring of the human lung microbiome. mBio. 2015;6:e02284-14.

14. Pragman AA, Kim HB, Reilly CS, Wendt C, Isaacson RE. The lung microbiome in moderate and severe chronic obstructive pulmonary disease. PLoS One. 2012;7:e47305.

15. Pragman AA, Lyu T, Baller JA, Gould TJ, Kelly RF, Reilly CS, et al. The lung tissue microbiota of mild and moderate chronic obstructive pulmonary disease. Microbiome. 2018;6:7.

16. Lozupone C, Cota-Gomez A, Palmer BE, Linderman DJ, Charlson ES,

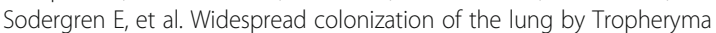
whipplei in HIV infection. Am J Respir Crit Care Med. 2013;187:1110-7.

17. Dickson RP, Erb-Downward JR, Freeman CM, McCloskey L, Beck JM, Huffnagle GB, et al. Spatial variation in the healthy human lung microbiome and the adapted island model of lung biogeography. Ann Am Thorac Soc. 2015;12:821-30.

18. Dickson RP, Erb-Downward JR, Freeman CM, McCloskey L, Falkowski NR, Huffnagle GB, et al. Bacterial topography of the healthy human lower respiratory tract. mBio. 2017;8:e02287-16. 
19. Segal LN, Clemente JC, Tsay J-CJ, Koralov SB, Keller BC, Wu BG, et al. Enrichment of the lung microbiome with oral taxa is associated with lung inflammation of a Th17 phenotype. Nat Microbiol. 2016;1:16031.

20. Youssef N, Sheik CS, Krumholz LR, Najar FZ, Roe BA, Elshahed MS. Comparison of species richness estimates obtained using nearly complete fragments and simulated pyrosequencing-generated fragments in $165 \mathrm{rRNA}$ gene-based environmental surveys. Appl Environ Microbiol. 2009;75:5227-36.

21. Liu Z, Lozupone C, Hamady M, Bushman FD, Knight R. Short pyrosequencing reads suffice for accurate microbial community analysis. Nucleic Acids Res. 2007;35:e120.

22. Wang Q, Garrity GM, Tiedje JM, Cole JR. Naiive Bayesian classifier for rapid assignment of rRNA sequences into the new bacterial taxonomy. Appl Environ Microbiol. 2007;73:5261-7.

23. Liu Z, DeSantis TZ, Andersen GL, Knight R. Accurate taxonomy assignments from 165 rRNA sequences produced by highly parallel pyrosequencers. Nucleic Acids Res. 2008;36:e120.

24. Kozich JJ, Westcott SL, Baxter NT, Highlander SK, Schloss PD. Development of a dual-index sequencing strategy and Curation pipeline for analyzing amplicon sequence data on the MiSeq Illumina sequencing platform. Appl Environ Microbiol. 2013;79:5112-20.

25. Callahan BJ, McMurdie PJ, Rosen MJ, Han AW, Johnson AJA, Holmes SP. DADA2: high-resolution sample inference from Illumina amplicon data. Nat Methods. 2016;13:581.

26. Grønseth R, Haaland I, Wiker HG, Martinsen EMH, Leiten EO, Husebø G, et al. The Bergen COPD microbiome study (MicroCOPD): rationale, design, and initial experiences. Eur Clin Respir J. 2014:1.

27. Bokulich NA, Subramanian S, Faith JJ, Gevers D, Gordon Jl, Knight R, et al. Quality-filtering vastly improves diversity estimates from Illumina amplicon sequencing. Nat Methods. 2013;10:57-9.

28. Callahan BJ, McMurdie PJ, Holmes SP. Exact sequence variants should replace operational taxonomic units in marker-gene data analysis. ISME J. 2017;11:2639-43.

29. Jousset A, Bienhold C, Chatzinotas A, Gallien L, Gobet A, Kurm V, et al. Where less may be more: how the rare biosphere pulls ecosystems strings. ISME J. 2017;11:853-62.

30. Sogin ML, Morrison HG, Huber JA, Welch DM, Huse SM, Neal PR, et al. Microbial diversity in the deep sea and the underexplored "rare biosphere.". PNAS. 2006;103:12115-20.

31. Berry D, Mahfoudh KB, Wagner M, Loy A. Barcoded primers used in multiplex amplicon pyrosequencing Bias amplification. Appl Environ Microbiol. 2011;77:7846-9.

32. Davis NM, Proctor DM, Holmes SP, Relman DA, Callahan BJ. Simple statistical identification and removal of contaminant sequences in marker-gene and metagenomics data. Microbiome. 2018;6:226.

33. Chandler DP, Fredrickson JK, Brockman FJ. Effect of PCR template concentration on the composition and distribution of total community 165 rDNA clone libraries. Mol Ecol. 1997;6:475-82.

34. Polz MF, Cavanaugh CM. Bias in template-to-product ratios in multitemplate PCR. Appl Environ Microbiol. 1998;64:3724-30.

35. Kennedy K, Hall MW, Lynch MDJ, Moreno-Hagelsieb G, Neufeld JD. Evaluating Bias of Illumina-based bacterial 165 rRNA gene profiles. Appl Environ Microbiol. 2014;80:5717-22.

36. Grønseth R, Drengenes C, Wiker HG, Tangedal S, Xue Y, Husebø GR, et al. Protected sampling is preferable in bronchoscopic studies of the airway microbiome. ERJ Open Res. 2017;3:00019-02017.

37. Segal LN, Alekseyenko AV, Clemente JC, Kulkarni R, Wu B, Chen H, et al. Enrichment of lung microbiome with supraglottic taxa is associated with increased pulmonary inflammation. Microbiome. 2013;1:19.

38. Rognes T, Flouri T, Nichols B, Quince C, Mahé F. VSEARCH: a versatile open source tool for metagenomics. PeerJ. 2016;4. https://doi.org/10.7717/peerj. 2584

\section{Publisher's Note}

Springer Nature remains neutral with regard to jurisdictional claims in published maps and institutional affiliations.

Ready to submit your research? Choose BMC and benefit from:

- fast, convenient online submission

- thorough peer review by experienced researchers in your field

- rapid publication on acceptance

- support for research data, including large and complex data types

- gold Open Access which fosters wider collaboration and increased citations

- maximum visibility for your research: over $100 \mathrm{M}$ website views per year

At BMC, research is always in progress.

Learn more biomedcentral.com/submissions 